\title{
The Severi bound on sections of rank two semistable bundles on a Riemann surface
}

\author{
By Javier Cilleruelo and Ignacio Sols*
}

\begin{abstract}
Let $E$ be a semistable, rank two vector bundle of degree $d$ on a Riemann surface $C$ of genus $g \geq 1$, i.e. such that the minimal degree $s$ of a tensor product of $E$ with a line bundle having a nonzero section is nonnegative. We give an analogue of Clifford's lemma by showing that $E$ has at most $(d-s) / 2+\delta$ independent sections, where $\delta$ is 2 or 1 according to whether the Krawtchouk polynomial $K_{r}(n, N)$ is zero or not at $r=(d-s) / 2+1, n=g, N=2 g-s$ (the analogous bound for nonsemistable rank two bundles being stronger but easier to prove). This gives an answer to the problem posed by Severi asking for the minimal degree of a directrix of a ruled surface. In some cases, namely if $s$ has maximal value $s=g$, or if $s \geq$ gonality $(C)-2$, or if $E$ is general among those of the same Segre invariant $s$, or also if the genus is a power of two, we prove the bound holds with $\delta=1$.

The theory of Krawtchouk polynomials investigates which triples $(g, s, d)$ provide zeros of $K_{r}(n, N)$. Then, they generate invariants which one may expect to be associated to a Severi bundle, i.e., to a rank two semistable bundle reaching the bound with $\delta=2$. According to this theory, there are only a finite number of such triples $(g, s, d)$ for each value of $d-s$, with the exception that there are infinitely many triples with $d-s=2$ or 4 . We then find all the Severi bundles corresponding to those two exceptional values of $d-s$.
\end{abstract}

\section{Introduction}

Let $C$ be a Riemann surface of genus $g \geq 1$ and let $E$ be a rank two vector bundle of degree $d$ on $C$. Then its Segre invariant $s$, which satisfies $s \equiv d \quad(\bmod 2), 2-2 g \leq s \leq g$, is the minimal degree of a twist $E \otimes L^{-1}$

*We thank David Butler for a personal communication which we have used in proving Proposition 3.1. We also thank Enrique Arrondo, Rafael Hernández, Jorge Jiménez and Bernardo López for conversations during the preparation of this article. 
having a nonzero section or, equivalently, the integer $s$ such that the maximal degree of a line subbundle $L \hookrightarrow E$ is $\frac{d-s}{2}$. In [16] Nagata proved that such an integer $s$ is at most $g$ and that we can assume $s \geq 2-2 g$, because otherwise $H^{1}\left(L^{*} \otimes(E / L)\right)=0$, which implies that $E$ is decomposable and the rank one Clifford bound is enough to bound its sections. Let us recall, furthermore, that the bundle $E$ is stable if $s>0$, and semistable if $s \geq 0$. If $E$ is special, i.e., if $h^{0}(E)$ is neither zero nor given by the Riemann-Roch formula $h^{0}(E)=$ $d+2-2 g$, then we have $s \leq d \leq 4 g-4-s$. Therefore we center our study in this "Severi range" of values of $d$. This study is trivial in case $E$ is not semistable, providing then a sharp bound (stronger than the "Severi bound" for semistable bundles in Theorem 0.2 below):

Proposition 0.1. Let $E$ be a rank two bundle of Segre invariant $s$, $2-2 g \leq s<0$, and degree $d$ on a Riemann surface of genus $g$.

$$
\begin{aligned}
& \text { If } s \leq d<-s \text { then } h^{0}(E) \leq\left[\frac{d-s}{4}\right]+1 . \\
& \text { If }-s \leq d \leq 4 g-4+s \text { then } h^{0}(E) \leq \begin{cases}\frac{d}{2}+\frac{3}{2} & \text { if } d \text { is odd } \\
\frac{d}{2}+2 & \text { if } d \text { is even, and } d \equiv s(4) \\
\frac{d}{2}+1 & \text { if } d \text { is even, and } d \equiv \equiv(4) .\end{cases} \\
& \text { If } 4 g-4+s<d \leq 4 g-4-s \text { then } h^{0}(E) \leq\left[\frac{3 d-s}{4}\right]-g+2 .
\end{aligned}
$$

Furthermore, for each $g, s, d$ in this range, there are a Riemann surface of genus $g$, and a rank two bundle of invariant $s$ and degree $d$ reaching the bound.

Indeed, by Serre duality it is enough to show the bound in the case $d \leq$ $2 g-2$. Let $L$ be a subbundle of maximal degree

$$
0 \longrightarrow L \longrightarrow E \longrightarrow L^{\prime} \longrightarrow 0
$$

i.e. $\operatorname{deg} L=\frac{d-s}{2} \leq 2 g-2$ and $\operatorname{deg} L^{\prime}=\frac{d-s}{2} \leq g-2$. Then the desired bound follows from $h^{0}(L) \leq\left[\frac{d-s}{4}\right]+1$ and $h^{0}\left(L^{\prime}\right) \leq \max \left\{0,\left[\frac{d+s}{4}\right]+1\right\}$ (for the case $-s \leq d \leq 2 g-2$, we note that $d+s \equiv d-s$ or $d+s \not \equiv d-s \quad(\bmod 4)$ depending on whether $d$ is even or odd). For the second assertion, since our assumption on the invariant $s$ implies that $g \geq 2$, we can take $C$ to be a hyperelliptic curve, and $E=L \oplus L^{\prime}$, where $\operatorname{deg} L=\frac{d-s}{2}, \operatorname{deg} L^{\prime}=\frac{d+s}{2}$ and $h^{0}(L)=\left[\frac{d-s}{4}\right]+1$, $h^{0}\left(L^{\prime}\right)=\max \left\{0,\left[\frac{d+s}{4}\right]+1\right\}$.

In Section 1 we present the proof of our main theorem, in which the binary Krawtchouk polynomials $K_{r}(n, N)$ appear. Let us recall that these polynomials are defined, for each $r \geq 0$, by their generating function

$$
\sum_{r} K_{r}(n, N) z^{r}=(1-z)^{n}(1+z)^{N-n} .
$$


TheOREm 0.2. Let E be a semistable rank two vector bundle of Segre invariant $s$ and degree $d$ on a Riemann surface of genus $g \geq 1$. If $s \leq d \leq$ $4 g-4-s$, the "Severi bound" holds:

$$
h^{0}(E) \leq \frac{d-s}{2}+\delta, \quad \text { where } \delta= \begin{cases}1 & \text { if } K_{\frac{d-s}{2}+1}(g, 2 g-s) \neq 0 \\ 2 & \text { if } K_{\frac{d-s}{2}+1}(g, 2 g-s)=0 .\end{cases}
$$

Furthermore, for each $g \geq 1,0 \leq s \leq g$ and $s \leq d \leq 4 g-4-s$, there are a Riemann surface of genus $g$, and a rank two bundle of invariant $s$ and degree $d$ having $\frac{d-s}{2}+1$ independent sections.

The "strict Severi bound" $h^{0}(E) \leq \frac{d-s}{2}+1$ was obtained by Arrondo and the second author [2], using different techniques, in the case of hyperelliptic curves. In fact, in [2] the strict Severi bound was conjectured, but Tan [22] constructed a counterexample with $g=3, s=2, d=4$, and $h^{0}=3$, i.e. his bundle reaches the Severi bound with $\delta=2$. We will refer to that case by saying that we have a "Severi bundle". In [9] Grzegorczyk, using an existence (nonconstructive) result of Teixidor i Bigas [23], proved the existence of a rank two bundle on a curve of genus $g=5$ having invariants $s=4, d=8$ and $h^{0}(E)=4$; thus it is also a Severi bundle.

Both Tan [21] and Arrondo-Sols [2] have remarked that an obvious duality argument allows one to show the Clifford bound $h^{0}(E) \leq \frac{d}{2}+2$ for a rank two semistable bundle in this range: Just apply the rank one Clifford lemma to $L, L^{\prime}$ in an extremal sequence as in proof of Proposition 0.1, observing again that $L, L^{\prime}$ can be assumed to be in the rank one Clifford range $(0 \leq$ degree $\leq 2 g-2)$ because, by Serre duality, the bundle $E$ can be assumed in its half Clifford range: $0 \leq d \leq 2 g-2$. Furthermore, it is implicitly shown in [2] that this bound can be reached only by $\mathcal{O}_{C} \oplus \mathcal{O}_{C}, \omega_{C} \oplus \omega_{C}$ for any curve $C$, and by $L \oplus L$ for a hyperelliptic curve and the line bundle $L$ of degree $d / 2$ and $h^{0}(L)=\frac{d}{4}+1$, assuming $d$ is a multiple of four (just combine Prop. 2 and Prop. 3 of [2] with the obvious remark that a nonsplit extension $0 \longrightarrow \mathcal{O}_{C} \longrightarrow \mathcal{E} \longrightarrow \mathcal{O}_{C} \longrightarrow 0$ in a hyperelliptic curve has $h^{0}(\mathcal{E})=1$, since $\mathbb{C}=H^{0}\left(\mathcal{O}_{C}\right) \longrightarrow H^{1}\left(\mathcal{O}_{C}\right)$ is the extension itself). The bound given in Theorem 0.2 is a refinement of this obvious bound and provides the analogue, in the theory of rank two bundles, to the Clifford bound in the theory of line bundles (the rank two Clifford bound $h^{0}(E) \leq \frac{d}{2}+2$ gives the analogue only in the case $s=0$ ).

In the Brill-Noether theory of special line bundles, it has been proved the nonemptiness [4] of the variety $W_{d}^{r}$ of those having degree $d$ and at least $r+1$ sections, if the expected dimension $\rho$ of this variety is nonnegative, i.e. if $(d, r)$ lies under some hyperbola refining the Clifford bound. It has also been proved that, if $\rho$ is positive, then $W_{d}^{r}$ is connected [6], and irreducible if the curve is general. The above Clifford lemma concerning rank two has motivated research of the analogous problems for bundles of rank two, starting 
from [23]. Our Severi refinement of the bound suggests a refinement of BrillNoether theory, now involving also the Segre invariant $s$, which amounts to the study of the intersections of the Brill-Noether strata with the irreducible strata corresponding to fixed values of $s$.

Finally, let us explain the reason why we name this bound and the corresponding bundles after Severi. It is due to the fact that it provides an answer to a problem posed by the Italian geometer, in "sulla clasificazione delle rigate algebrice" [17], for a ruled surface $S$ of $\mathbb{P}^{r}$ of degree $d$ and irregularity $q$, which he always assumed to be a linearly normal scroll. After remarking that the minimal degree $\mu$ of a directrix of $S$ is at most $d-[r / 2]$, he proposed as an open problem to find upper bounds of $\mu$ below this obvious one. If $E$ is the rank two vector bundle of degree $d$ on a curve of genus $q$ such that $S=\mathbb{P}(E)$ and $H^{0}\left(\theta_{S}(1)\right)=H^{0}(E)$ (so that $\mathbb{P}^{r}$ is $\mathbb{P}\left(H^{0}(E)\right)$ ), then $\mu$ is related to the Segre invariant $s$ of $E$ as $\mu=\frac{d+s}{2}$, so that our bounds in Proposition 0.1 and Theorem 0.2 translate into upper bounds for $\mu$. For instance, the bound in our main Theorem 0.2 , for special bundles with $s \geq 0$, translates as that linearly normal scrolls of $\mathbb{P}^{r}$ whose irregularity $q$ is not given by the Riemann-Roch formula $r=d+1-2 q$, and such that $\mu \geq d / 2$, have $\mu$ and $d, r$ related by

$$
\mu \leq d-r+\delta-1,
$$

where $\delta=1$ or 2 according to whether $K_{r}(q, 2 q-2 \mu+d)$ is zero or not. Analogous bounds, corresponding to the rest of the cases $(\mu<d / 2)$, are obtained as translation of Proposition 0.1 in terms of ruled surfaces.

As for the content of the article, in Section 1 we show Theorem 0.2 proving the main Lemma 1.1 which asserts that a rank two bundle, having at least $r<g$ independent sections, must have a section vanishing at least at $r$ points, unless $K_{r}(g, 2 g+2 r-2-d)=0$. Then we prove recursively that this equality cannot be fulfilled, when fixing $g, r$, by two consecutive values of $d$. We believe that other lemmas of this kind should work in algebraic geometry, namely, if a coherent sheaf has many sections, then some section should fulfill a given property, provided that the data do not satisfy some arithmetical relation.

Section 2 is devoted to the strict Severi bound. We prove it for $s=1$, $s=g$ and for other values of the invariants, for the general bundle on any curve, and also whenever $s \leq n-2$, where $n$ is the gonality of the curve (recall $n \leq \frac{g}{2}+1$, for the general curve of genus $g$ ).

The study of the integral zeros of the Krawtchouk polynomials, a subject under current research, allows us to find the values $g, s, d$ for which Severi bundles, i.e. rank two bundles $E$ with $h^{0}(E)=\frac{d-s}{2}+2$, are expectable. This is a rather striking fact, because in a different research area, the Krawtchouk polynomials appear as the polynomials associated to Hamming schemes, and their integral zeros are then related with pathologies in coding theory, graph theory and the Radon Transform on $\mathbb{Z}_{2}^{n}$ [13]. Perhaps our bound might find 
some applications in this field, since in a recent, still unpublished, work of T. Johnsen [12] has interpreted errors in coding theory as rank two bundles on a Riemann surface, making essential use of the Segre invariant $s$, as "syndrome" of a Goppa code.

In particular, no Severi bundles exist when $s<0$ and the only one with $s=0$ is the trivial one. There are no Severi bundles when $g$ is a power of two. For $d=s$, there are no Severi bundles other than $\mathcal{O}_{C} \oplus \mathcal{O}_{C}, \omega_{C} \oplus \omega_{C}$. For hyperelliptic curves, we must have $E=L \oplus L$, where $L$ is the hyperelliptic bundle of degree $\frac{d}{2}$ (under the assumption that $d$ is a multiple of 4). For $d=s+2, s+4$ there are infinitely many triples $(g, s, d)$ for which Severi bundles are expectable, the genus $g$ being the one of plane curves, $g=\frac{(d-1)(d-2)}{2}$, in the first case, and being in the second case the maximal genus $g=\frac{(d-2)(d-3)}{6}$ of nondegenerate curves of $\mathbb{P}^{4}$ of degree $d \not \equiv 1 \quad(\bmod 3)$. We find in Section 3 all such bundles: Those with $d=s+2$ are the restrictions of $T_{\mathbb{P}^{2}}(-1)$ to plane curves. Among the triples with $d=s+4$, only $(5,4,8)$ corresponds to Severi bundles, and these are the stable restrictions of the universal bundle of $\operatorname{Gr}(2,4)$ to curves of genus 5 and bidegree $(6,2)$ contained in special linear complexes. For each value $d>s+4$, only for finitely many triples $(g, s, d)$ one can expect the existence of Severi bundles. Actually, all the possible triples for $s-d \leq 12$ are known (i.e. the zeros of the corresponding Krawtchouck polynomials are known at present), and we list them to stimulate the search of such sporadic bundles.

\section{The Severi bound}

The Krawtchouk polynomials $K_{r}(n, N)$ defined by

$$
\sum_{r=0}^{\infty} K_{r}(n, N) z^{r}=(1-z)^{n}(1+z)^{N-n}
$$

satisfy the following relations [13]:

$$
\begin{aligned}
K_{r}(n, N) & =(-1)^{r} K_{r}(N-n, N), \\
K_{r}(n, N) & =(-1)^{r} K_{N-r}(n, N), \\
\left(\begin{array}{c}
N \\
n
\end{array}\right) K_{r}(n, N) & =\left(\begin{array}{c}
N \\
r
\end{array}\right) K_{n}(r, N), \\
K_{r}(n, N) & =\sum_{j=0}^{r}(-1)^{j}\left(\begin{array}{c}
n \\
j
\end{array}\right)\left(\begin{array}{c}
N-n \\
r-j
\end{array}\right), \\
K_{r}(n, N) & =\sum_{j=0}^{r}(-2)^{j}\left(\begin{array}{c}
n \\
j
\end{array}\right)\left(\begin{array}{c}
N-j \\
r-j
\end{array}\right) .
\end{aligned}
$$


The key for the proof of Theorem 0.2 is the following

Lemma 1.1. Let $E$ be a rank two bundle of degree $d$ of a curve $C$ of genus $g$ having at least $r+1 \leq g$ independent sections. There is a nonzero section of $E$ vanishing at $r$ points, provided that $K_{r}(g, 2 g+2 r-2-d) \neq 0$.

Proof. Let $\Gamma=p_{1}+\cdots+p_{m}$ be a divisor of $C$ of degree $m \gg 0$ so that $E^{*} \otimes \omega_{C}(\Gamma)$ is generated by global sections. For a general choice of two sections, we get a sequence

$$
0 \longrightarrow \mathcal{O}_{C}^{2} \longrightarrow E^{*} \otimes \omega_{C}(\Gamma) \longrightarrow \mathbb{C}\left(q_{1}\right) \oplus \cdots \oplus \mathbb{C}\left(q_{4 g-4+2 m-d}\right) \longrightarrow 0 \quad\left(q_{j} \in C\right) .
$$

Let $I=\left\{(p, D) \in\left(C \times C_{r}\right) \mid D-p \geq 0\right\} \subseteq C \times C_{r}$ be the incidence diagram associated to the $r$-symmetric power $C_{r}$, and $\pi_{1}, \pi_{2}$ the obvious projections

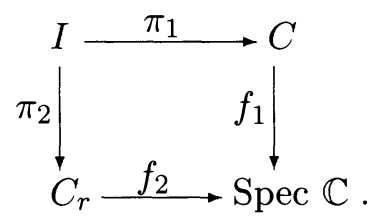

Applying the functor $\pi_{2 *} \pi_{1}^{*}$ to the sequences

$$
\begin{gathered}
0 \longrightarrow E \longrightarrow \omega_{C}^{2}(\Gamma) \longrightarrow \mathbb{C}\left(q_{1}\right) \oplus \cdots \oplus \mathbb{C}\left(q_{4 g-4+2 m-d}\right) \longrightarrow 0, \\
0 \longrightarrow \omega_{C}^{2} \longrightarrow \omega_{C}^{2}(\Gamma) \longrightarrow \mathbb{C}\left(p_{1}\right)^{2} \oplus \cdots \oplus \mathbb{C}\left(p_{m}\right)^{2} \longrightarrow 0,
\end{gathered}
$$

we obtain

$$
\begin{aligned}
& 0 \longrightarrow \pi_{2 *} \pi_{1}^{*} E \longrightarrow \pi_{2 *} \pi_{1}^{*} \omega_{C}^{2}(\Gamma) \longrightarrow \bigoplus \mathcal{O}_{X_{q_{j}}} \longrightarrow 0 \\
& 0 \longrightarrow \pi_{2 *} \pi_{1}^{*} \omega_{C}^{2} \longrightarrow \pi_{2 *} \pi_{1}^{*} \omega_{C}^{2}(\Gamma) \longrightarrow \bigoplus \mathcal{O}_{X_{p_{i}}}^{2} \longrightarrow 0,
\end{aligned}
$$

where $X_{p}$ is the divisor $\left\{D \in C_{r} \mid D-p \geq 0\right\}$ of $C_{r}$. Obviously $X_{p}$ is algebraically equivalent to $X_{q}$, for all $p, q \in C$. Let us denote by $x$ the algebraic class of such divisors.

The Chern polynomial of $\pi_{2 *} \pi_{1}^{*}\left(\omega^{2}\right)=\left(\pi_{2 *} \pi_{1}^{*} \omega\right)^{2}=\Omega_{C_{r}}^{2}$ (cf. [1, IV, Lemma 2.3]) may be computed from the well-known formula

$$
c_{t}\left(T_{C_{r}}\right)=(1+x t)^{r-g+1} e^{\frac{-\theta t}{1+x t}},
$$

where $\theta$ is the pull-back of the class of the theta divisor of the Jacobian variety $J C$ of $C$ by the Abel-Jacobi map $C_{r} \longrightarrow J C$ (cf. [1, VII $\S 5$, formula (5.4) and subsequent remark]). Therefore,

$$
c_{t}\left(\Omega_{C_{r}}\right)^{2}=c_{-t}\left(T_{C_{r}}\right)^{2}=(1-x t)^{2(r-g+1)} e^{\frac{2 \theta t}{1-x t}} .
$$


On the other hand, $c_{t}\left(\mathcal{O}_{X_{p_{i}}}\right)$ has Chern polynomial $c_{t}\left(\mathcal{O}\left(-X_{p_{i}}\right)\right)^{-1}=$ $(1-x t)^{-1} ;$ thus,

$$
\begin{aligned}
c_{t}\left(\pi_{2 *} \pi_{1}^{*} \omega^{2}(\Gamma)\right) & =(1-x t)^{2(r-g+1)-2 m} e^{\frac{2 \theta t}{1-x t}} \\
c_{t}\left(\pi_{2 *} \pi_{1}^{*} E\right) & =(1-x t)^{2 r+2 g-2-d} e^{\frac{2 \theta t}{1-x t}} .
\end{aligned}
$$

Applying $f_{1_{*}}$ to the unit $E \longrightarrow \pi_{1 *} \pi_{1}^{*} E$ of the adjunction of functors $\pi_{1}^{*} \dashv \pi_{1 *}$ we get

$$
f_{1 *} E \longrightarrow f_{1_{*}} \pi_{1 *} \pi_{1}^{*} E=f_{2_{*}} \pi_{2 *} \pi_{1}^{*} E
$$

thus, equivalently,

$$
e v_{r}: \mathbb{C}^{r+1} \otimes \mathcal{O}_{C_{r}} \hookrightarrow f_{2}^{*} f_{1 *} E=H^{0}(E) \otimes \mathcal{O}_{C_{r}} \longrightarrow \pi_{2 *} \pi_{1}^{*} E .
$$

For a point $D \in C_{r}$, i.e. for a divisor $D$ of $C$ of degree $r$, the linear map

$$
e v_{r}(D): \mathbb{C}^{r+1} \hookrightarrow H^{0}(E) \longrightarrow H^{0}\left(E \otimes \mathcal{O}_{D}\right)
$$

is clearly the "evaluation" map. The locus $X_{r}\left(e v_{r}\right)$ where this map has rank at most $r$ has expected dimension $r-((r+1)-r)(2 r-r)=0$, and Porteous formula [1] tells us that $X_{r}\left(e v_{r}\right)$ has degree $c_{r}\left(\pi_{2 *} \pi_{1}^{*} E\right) \in A^{r}\left(C_{r}\right) \simeq \mathbb{Z}$ if it is empty or it has the expected dimension.

If $c_{r}\left(\pi_{2 *} \pi_{1}^{*} E\right) \neq 0$, then $X_{r}\left(e v_{r}\right)$ cannot be empty, since the Porteous lemma would then apply and it would assert its degree is $c_{r}\left(\pi_{2 *} \pi_{1}^{*} E\right)$, yielding a contradiction. For $D \in X_{r}\left(e v_{r}\right) \subset C_{r}$, the evaluation map $e v_{r}(D)$ has nontrivial Kernel, i.e. there is a nonzero section of $E$ vanishing at $D$, as wanted. Therefore, we will end the proof showing our claim that

$$
c_{r}\left(\pi_{2 *} \pi_{1}^{*} E\right)=(-1)^{r} K_{r}(g, 2 g+2 r-2-d) .
$$

Because of Poincaré's formula ([1, Chap 1, §5]), and the projection formula applied to the Abel-Jacobi map, together with the fact that $\theta^{g}=g$ ! in $J C$, it is $\theta^{k} x^{r-k}=k !\left(\begin{array}{l}g \\ k\end{array}\right)$; thus, in particular, $x^{r}=1$ (as used, for instance, in the proof of Proposition 4.2 [1]). The proof of the claim starts with a computation which uses twice the identity $\frac{1}{(1-z)^{k}}=\sum_{j}\left(\begin{array}{l}j-1 \\ k-1\end{array}\right) z^{j-k}$ :

$$
\begin{aligned}
e^{\frac{2 \theta t}{1-x t}} & =x^{r} \sum_{k} \frac{(2 \theta t)^{k}}{k !} \frac{1}{(1-x t)^{k}} \\
& =x^{r} \sum_{k} \frac{(2 \theta t)^{k}}{k !} \sum_{j}\left(\begin{array}{l}
j-1 \\
k-1
\end{array}\right)(x t)^{j-k}=\sum_{k, j} \frac{1}{k !}\left(\begin{array}{l}
j-1 \\
k-1
\end{array}\right) 2^{k}(x t)^{j} \theta^{k} x^{r-k} \\
& =\sum_{k} 2^{k}\left(\begin{array}{l}
g \\
k
\end{array}\right) \sum_{j}\left(\begin{array}{l}
j-1 \\
k-1
\end{array}\right)(x t)^{j}=\sum_{k} 2^{k}\left(\begin{array}{l}
g \\
k
\end{array}\right) \frac{(x t)^{k}}{(1-x t)^{k}} \\
& =\left(1+\frac{2 x t}{1-x t}\right)^{g}=\frac{(1+x t)^{g}}{(1-x t)^{g}} .
\end{aligned}
$$


The wanted coefficient $c_{r}\left(\pi_{2 *} \pi_{1}^{*} E\right)$ of $t^{r}=(x t)^{r}$ in the polynomial

$$
c_{t}\left(\pi_{2 *} \pi_{1}^{*} E\right)=(1-x t)^{2 r+g-d-2}(1+x t)^{g}
$$

is just the coefficient of $t^{r}$ in the polynomial $(1+t)^{g}(1-t)^{g+2 r-2-d}$; i.e.

$c_{r}\left(\pi_{2 *} \pi_{1}^{*} E\right)=K_{r}(g+2 r-2-d, 2 g+2 r-2-d)=(-1)^{r} K_{r}(g, 2 g+2 r-2-d)$,

by (1.1) and (1.2).

Lemma 1.2. When $N \geq n, r$ are nonnegative integers, then there is no identity $K_{r}(n, N)=K_{r}(n, N+1)=0$.

Proof. Let $p(z)=(1-z)^{n}(1+z)^{N-n}=\sum_{r} K_{r}(n, N) n^{r}$, and let us take the logarithmic derivative $\frac{p^{\prime}(z)}{p(z)}=-\frac{n}{1-z}+\frac{N-n}{1+z}$. Thus, $\left(1-z^{2}\right) p^{\prime}(z)=$ $(N-2 n-z N) p(z)$. Identifying coefficients, we obtain the following recurrence relation for $K_{r}=K_{r}(n, N)$ :

$$
\begin{gathered}
r K_{r}=(N-2 n) K_{r-1}+(r-N-2) K_{r-2}, \quad r \geq 2 \\
\quad \text { and } \quad K_{0}=1, \quad K_{1}=N-2 n .
\end{gathered}
$$

Therefore, if two consecutive $K_{r}, K_{r-1}, 1 \leq r \leq N$, vanish at $n, N$, it is also the case for $K_{r-2}$ and thus the process iterates to conclude $K_{0}=0$, yielding a contradiction. Thus we have proved that, for all nonnegative integers $n, r, N$ with $n, r \leq N$, it is $K_{r}(n, N) \neq 0$ or $K_{r-1}(n, N) \neq 0$.

Now, assume that $K_{r}(n, N)=K_{r}(n, N+1)=0$. The identity

$$
(1-z)^{n}(1+z)^{N+1-n}=(1-z)^{n}(1+z)^{N-n}(1+z)
$$

gives the relation $K_{r}(n, N+1)=K_{r}(n, N)+K_{r-1}(n, N)$, and then $K_{r-1}(n, N)=$ $K_{r}(n, N)=0$, which is impossible.

Proof of Theorem 0.2. By the Serre duality theorem, it is enough to show the theorem in half of the Severi range $s \leq d \leq 2 g-2$. In order to prove the bound we distinguish two cases:

Case $s=0$. Then the theorem asserts that $h^{0}(E) \leq \frac{d}{2}+1$ unless $d$ is a multiple of four. But this has been already proved (recall [21] and [2]) in the introduction.

Case $s>0$. Denote $r=\frac{d-s}{2}+1 \leq g-1$. Assume first that $K_{r}(g$, $2 g-s) \neq 0$. If $E$ had $r+1$ independent sections, then applying Lemma 1.1 to $E$ we would obtain a nonzero section of $E$ vanishing at least at $r$ points, thus a nonzero section of a twist of $E$ of degree $s-2$, which is impossible. Therefore, $E$ actually satisfies the strict Severi bound $h^{0}(E) \leq \frac{d-s}{2}+1$. 
Now assume $K_{r}(g, 2 g-s)=0$, and thus, by Lemma $1.2, K_{r}\left(g, 2 g-s^{\prime}\right) \neq 0$ for the consecutive values $s^{\prime}=s \pm 1$. Take an elementary transform of $E$

$$
0 \longrightarrow E^{\prime} \longrightarrow E \longrightarrow \mathbb{C}(p) \longrightarrow 0
$$

of degree $d^{\prime}=d-1$ and Segre invariant $s^{\prime}=s \pm 1$. Since $r$ is $\frac{d^{\prime}-s^{\prime}}{2}+1$, or $\frac{d^{\prime}-s^{\prime}}{2}+2$ and $K_{r}\left(g, 2 g-s^{\prime}\right) \neq 0$, Lemma 1.1 implies $h^{0}\left(E^{\prime}\right) \leq r$; otherwise $E^{\prime}$ would have a nonzero section vanishing at $r$ sections, thus yielding a contradiction. Therefore $h^{0}(E) \leq r+1$, as wanted.

As for the last assertion of the theorem, we can assume $g \geq 2$, because if $g=1$, then $d=s=0$ and $\mathcal{O}_{C} \oplus \mathcal{O}_{C}$ satisfies the requirement. Consider a hyperelliptic Riemann surface $C$ of genus $g$. Take general points $p, p_{1}, \ldots, p_{s+1} \in C$. The general elementary transform

$$
0 \longrightarrow F \longrightarrow \mathcal{O}_{C} \oplus \mathcal{O}_{C}(-p) \longrightarrow \mathbb{C}\left(p_{1}\right) \oplus \cdots \oplus \mathbb{C}\left(p_{s+1}\right) \longrightarrow 0
$$

is a rank two bundle $F$ of invariant $s$ and degree $-s-2$. For $q, q^{\prime}$, an involutive pair of points of $C$, consider the divisor $D=\left(\frac{d+s+2}{4}\right)\left(q+q^{\prime}\right)$ or $\left(\frac{d+s}{4}\right)\left(q+q^{\prime}\right)$ according to whether $d+s \equiv 2$ or $0 \quad(\bmod 4)$. In the first case $E=F(D)$ is a rank two bundle of Segre invariant $s$, degree $d$, and

$$
h^{0}(E) \geq h^{0}(D)+h^{0}(D-p)-(s+1)=\frac{d-s}{2}+1 .
$$

In the second case, the rank two bundle $E=F(D+p)$ has Segre invariant $s$, degree $d$, and

$$
h^{0}(E) \geq h^{0}(D+p)+h^{0}(D)-(s+1)=\frac{d-s}{2}+1 .
$$

This is actually an equality because of Proposition 3 of [2].

\section{The strict Severi bound}

It was proved by Arrondo and the second author [2] that, on a hyperelliptic curve, the strict Severi bound is satisfied by all rank two semistable bundles other than $\mathcal{O}_{C} \oplus \mathcal{O}_{C}$ and $\omega_{C} \oplus \omega_{C}$, and for each positive integer $d \equiv 0 \quad(\bmod 4)$, the direct sum $L \otimes L$, being $L$ the line bundle on $C$ having $\operatorname{deg} L=\frac{d}{2}$ and $h^{0}(L)=\frac{d}{4}+1$. (This is Prop. 3 of [2] combined with the obvious fact that $h^{0}(\mathcal{E})=1$ for any nonsplit extension $0 \longrightarrow \mathcal{O}_{C} \longrightarrow \mathcal{E} \longrightarrow \mathcal{O}_{C} \longrightarrow 0$.) We will now provide conditions under which the strict Severi bound holds for rank two bundles on nonhyperelliptic curves.

In the first part of this section, we will study the Krawtchouk triples, i.e. the values of $(g, s, d)$ in the Severi range, $0 \leq s \leq d \leq 4 g-4-s, s \leq g$, for which the Krawtchouk relation $K_{\frac{d-s}{2}+1}(g, 2 g-s)=0$ is fulfilled, thus obtaining, by 
purely arithmetical reasons the strict Severi bound for the rest of the values. In the second part we find large families of bundles which satisfy the strict Severi bound for geometrical reasons.

Remark. It is an easy consequence of properties (1.2) and (1.3) that the set of triples of integers $(r, n, N)$ satisfying $K_{r}(n, N)=0$ is closed under the action of a group of order eight generated by the involutions $(r, n, N) \leftrightarrow(r, N-n, N)$ and $(r, n, N) \leftrightarrow(n, r, N)$ [13]. If we take $r=\frac{d-s}{2}+1, n=g$ and $N=2 g-s$, the corresponding generating involutions are

$$
\begin{aligned}
& i_{1}:(g, s, d) \longrightarrow(g, s, 4 g-4-d), \\
& i_{2}:(g, s, d) \longrightarrow\left(2 g-\frac{d+s}{2}-1,2 g-d-2,4 g-4-d-2 s\right) .
\end{aligned}
$$

Observe that $i_{1}$ is given by Serre duality. We wonder if some kind of geometrical duality might also corresponds to involution $i_{2}$ (since this involution changes the genus $g$, it should change the curve, perhaps to another curve in $\mathbb{P}(E))$.

We are interested in the triples $(g, s, d)$ in the Severi range, but due to the symmetry of $i_{1}$ respect to $d=2 g-2$, we will consider, in the sequel, the triples in the half Severi range

$$
H S=\{(g, s, d) ; 0 \leq s \leq d \leq 2 g-2, \quad s \leq g\} .
$$

First of all, we study the triples in the boundaries of $H S: s=0, s=d$, $d=2 g-2, s=g$.

Lemma 2.1. The only Krawtchouk triples in the boundaries of HS are the families

$$
\begin{aligned}
& B_{1}=\{(g, 0, d), d \equiv 0 \quad(\bmod 4), 0 \leq d \leq 2 g-2\} \\
& B_{2}=\{(g, s, 2 g-2), g \text { odd, s even, } 0 \leq s \leq g\}
\end{aligned}
$$

Proof. We recall that $K_{\frac{d-s}{2}+1}(g, 2 g-s)$ is the coefficient of $z^{\frac{d-s}{2}+1}$ in $(1-z)^{g}(1+z)^{g-s}=(1-z)^{s}\left(1-z^{2}\right)^{g-s}$. If $s=0$, the coefficients vanish if and only if $z^{\frac{d+2}{2}}$ is an odd power of $z$, i.e. $d \equiv 0 \quad(\bmod 4)$. These triples form the family $B_{1}$.

If $s=d$, the coefficient of $z$ is $-s$. Thus, these triples $(g, 0,0)$ are contained in $B_{1}$.

If $s=g$, all the coefficients of $(1-z)^{s}$ are different from zero, i.e. there are no Krawtchouk triples with $s=g$. 
For the boundary $d=2 g-2$, applying (1.4) and (1.3), we obtain $K_{g-\frac{s}{2}}(g$, $2 g-s) \neq 0$ if and only if $K_{g}\left(g-\frac{s}{2}, 2 g-s\right) \neq 0$, which is the coefficient of $z^{g}$ in $\left(1-z^{2}\right)^{g-\frac{s}{2}}$; thus it is nonzero if and only if $s$ is even and $g$ is odd. These triples form the family $B_{2}$.

Now, as a corollary of this lemma we can describe the invariants, in the boundary of the $H S$ range, of bundles for which the strict Severi bound is assured by arithmetical means.

COROllary 1. For a rank two bundle $E$ of invariant $s$ and degree $d$ with $0 \leq s \leq d \leq 2 g-2$, the strict Severi bound $h^{0}(E) \leq \frac{d-s}{2}+1$ holds in the cases:

i) $s=0 \quad d \not \equiv 0 \quad(\bmod 4)$,

ii) $s=g$,

iii) $s=d, \quad s>0$,

iv) $d=2 g-2, \quad g$ even.

Next we study the Krawtchouk triples in $H S$ which are not in the boundaries, depending on the values of $d-s$.

We consider the list

\begin{tabular}{|c|c|}
\hline$\frac{d-s}{2}=k$ & $F_{k}$ \\
\hline 1 & $\left(\frac{(d-2)(d-1)}{2}, d-2, d\right), \quad d \geq 5$ \\
\hline 2 & $\left(\frac{(d-3)(d-2)}{6}, d-4, d\right), \quad d \not \equiv 1 \quad(\bmod 3), \quad d \geq 9$ \\
\hline 3 & $(10,3,9)(36,6,12)(806,91,97)(7567,91,97)$ \\
\hline 4 & $(14,11,19)(22,8,16)(45,23,31)(39,11,19)$ \\
& $(156,23,31)(5590,298,306)$ \\
\hline 5 & $(14,12,22)(14,3,13)(67,5,15)(345,35,45)$ \\
\hline 6 & $(13,9,21)(19,16,28)(1029,37,49)$ \\
\hline$k \geq 7$ & Finite list for each $k$ \\
\hline
\end{tabular}

Lemma 2.2. For $k=1, \ldots, 6$ the family $F_{k}$ is complete and, for $k \geq 3$, it is finite.

Proof. We only have to prove that $F_{k}$ contains all the Krawtchouk triples $(g, s, d)$, in the range $0<s<d<2 g-2$, satisfying $\frac{d-s}{2}=k$.

For $d-s=2,4$, the value $K_{\frac{d-s}{2}+1}(g, 2 g-s)$ is computed from (1.5):

$$
\begin{aligned}
& K_{2}(g, 2 g-s)=\left(\begin{array}{c}
g-s \\
2
\end{array}\right)-g(g-s)+\left(\begin{array}{l}
g \\
2
\end{array}\right) \\
& K_{3}(g, 2 g-s)=\left(\begin{array}{c}
g-s \\
3
\end{array}\right)-\left(\begin{array}{c}
g-s \\
2
\end{array}\right) g+(g-s)\left(\begin{array}{l}
g \\
2
\end{array}\right)-\left(\begin{array}{l}
g \\
3
\end{array}\right) .
\end{aligned}
$$

Equating these expressions to zero, we obtain $F_{1}$ and $F_{2}$. 
For $d-s=6,8,10,12$, the equations $K_{\frac{d-s}{2}+1}(g, 2 g-s)=0$ are elliptic equations. The complete lists of the integral roots of $K_{r}$ with $r \leq 7$ have been found in the last years by different authors: $K_{4}([15],[18]), K_{5}([10]), K_{6}([19])$ and $K_{7}([20])$.

The second part of the lemma is a consequence of Theorem 2 in [13], where it is proved that, for fixed $r \geq 4$, there are finitely many integers $n, N$ such that $K_{r}(n, N)=0$.

The involution $i_{2}$ leads to another way of classifying Krawtchouk triples, depending on the values of $g-s$. We observe that the image of the equation $\frac{d-s}{2}=k$ by this involution is $g-s-1=k$. We again list the Krawtchouk triples which do not lie in the boundary:

\begin{tabular}{|c|l|}
\hline$g-s-1=k$ & \multicolumn{1}{|c|}{$F_{k}^{\prime}$} \\
\hline 1 & $\left(m^{2}-2, m^{2}-4,2 m^{2}-m-6\right), \quad m \geq 3$ \\
\hline 2 & $\left(\frac{m^{2}-7}{3}, \frac{m^{2}-16}{3}, \frac{2 m^{2}-3 m-20}{3}\right), m \neq \equiv \quad(\bmod 3), m \geq 5$ \\
\hline 3 & $\begin{array}{l}(13,9,22)(62,58,116)(1517,1513,2941) \\
(15039,15035,29985)\end{array}$ \\
\hline 4 & $\begin{array}{l}(12,7,11)(31,26,52)(62,57,99)(62,57,111) \\
(284,279,543)(10877,10872,21454)\end{array}$ \\
\hline 5 & $(10,4,6)(19,13,27)(123,117,239)(649,643,1261)$ \\
\hline 6 & $(10,3,9)(15,8,12),(2014,2007,3989)$ \\
\hline$k \geq 7$ & Finite list for each $k$ \\
\hline
\end{tabular}

COROllary 2. For each $k=1, \ldots, 6$, and rank two vector bundle $E$ on a curve of genus $g$, of Segre invariant $s$ and degree $d$ in the range $0<s<$ $d<2 g-2, s<g$, the strict Severi bound $h^{0}(E) \leq \frac{d-s}{2}+1$ holds, whenever $\frac{d-s}{2}=k($ resp. $g-s-1=k)$ and $(g, s, d) \notin F_{k}\left(\right.$ resp. $\left.(g, s, d) \notin F_{k}^{\prime}\right)$.

Proof. It is a consequence of Theorem 0.2 and Lemma 2.2 by application of the involution $i_{2}$.

It is also possible to say something about small positive values of $s$.

Proposition 2.3. For a rank two vector bundle $E$ on a curve of genus $g$, of Segre invariant $s$ and degree d, with $0 \leq s \leq d \leq 2 g-2$, the strict Severi bound $h^{0}(E) \leq \frac{d-s}{2}+1$ holds in the following cases:
i) $s=1$,
ii) $s=2$, with $d \neq 2 g-6$,
iii) $s=3$, with $d \neq g-1$ or $g \not \equiv 2 \quad(\bmod 4)$. 
Proof. $K_{\frac{d-s}{2}+1}(g, 2 g-s)$ is the coefficient of $z^{\frac{d+1}{2}}, z^{\frac{d}{2}}$ and $z^{\frac{d-1}{2}}$ in $(1-z)\left(1-z^{2}\right)^{g-1},(1-z)^{2}\left(1-z^{2}\right)^{g-2}$ and $(1-z)^{3}\left(1-z^{2}\right)^{g-3}$ respectively. Thus an easy computation yields i), ii) and iii).

Remark 2.4. We double the information given in the above propositions by recalling that, for $d \geq 2 g-2$, a triple $(g, s, d)$ satisfies the Krawtchouk relation if and only if $i_{1}(g, s, d)=(g, s, 4 g-4-d)$ does.

We finish this arithmetical part of our analysis with the following:

Proposition 2.5. For a rank two bundle $E$ of Segre invariant $s$ and degree $d$ with $0<s \leq d \leq 4 g-4-s$ on a curve whose genus $g$ is a power of two, the strict Severi bound $h^{0}(E) \leq \frac{d-s}{2}+1$ holds.

Proof. Let $r=\frac{d-s}{2}+1$; thus $1 \leq r \leq 2 g-s-1$. By (1.4) and (1.6) we have

$$
K_{r}(g, 2 g-s)=\frac{\left(\begin{array}{l}
2 g-s \\
r
\end{array}\right)}{\left(\begin{array}{c}
2 g-s \\
g
\end{array}\right)} K_{g}(2,2 g-s)=\frac{\left(\begin{array}{c}
2 g-s \\
r
\end{array}\right)}{\left(\begin{array}{c}
2 g-s \\
g
\end{array}\right)} \sum_{j=0}^{g}(-2)^{j}\left(\begin{array}{l}
r \\
j
\end{array}\right)\left(\begin{array}{c}
2 g-s-j \\
g-j
\end{array}\right) .
$$

The sum on the right-hand side has the same parity as $\left(\begin{array}{c}2 g-s \\ g\end{array}\right)$. On the other hand, it is easy to see that, if $g$ is a power of two and $s>0$, then $\left(\begin{array}{c}2 g-s \\ g\end{array}\right)$ is an odd number. Thus $K_{r}(g, 2 g-s) \neq 0$.

In the geometrical part of this section we prove first the strict bound in case $s$ is small. Recall that the gonality $n$ of a curve is the minimum integer such that the curve has a $g_{n}^{1}$. (It is known that the gonality of the general curve of genus $g$ is at most $\frac{g}{2}+1$.)

THEOREM 2.6. Let $E$ be a rank two bundle of Segre invariant $s>0$ and degree $d$, on a curve $C$ of genus $g \geq 1$ and gonality $n$. If $s \leq n-2$ and $s \leq d \leq 4 g-4-s$, then

$$
h^{0}(E) \leq \frac{d-s}{2}+1
$$

Proof. We can assume $g \geq 3$, since for $g=1,2$, it is $n=2$, so that $s>n-2$. Furthermore, we can assume, by Corollary 2.2, that $d>s$. Observe that, because of the Riemann-Roch theorem, to prove the bound is equivalent to prove $h^{0}(E)+h^{0}\left(E^{*} \otimes \omega_{C}\right) \leq 2 g-s$. Let

$$
0 \longrightarrow L \longrightarrow E \longrightarrow L^{\prime} \longrightarrow 0
$$

be, as in Section 1, a line subbundle of maximal degree $\frac{d-s}{2}$. Thus $\operatorname{deg} L^{\prime}=$ $\frac{d+s}{2}>\operatorname{deg} L$. We distinguish three cases.

Case $\frac{d+s}{2} \leq n-1$. Then $h^{0}(E) \leq h^{0}(L)+h^{0}\left(L^{\prime}\right)=1+1$. 
Case $\frac{d-s}{2} \leq n-1<\frac{d+s}{2}$. Choosing an effective divisor $D$ of degree $\frac{d+s}{2}-n+1>0$, we get

$$
\begin{aligned}
h^{0}(E) & \leq h^{0}(L)+h^{0}\left(L^{\prime}(-D)\right)+\operatorname{deg} D \\
& \leq 1+\left(1+\left(\frac{d+s}{2}-n+1\right)\right) \leq \frac{d-s}{2}+1 .
\end{aligned}
$$

Case $n \leq \frac{d-s}{2}$. In this case we give a proof analogous to the one provided in [2] by Arrondo and the second author for the case of hyperelliptic curves. Consider the subschemes

$$
\begin{aligned}
& X=\left\{M \in \operatorname{Pic}^{1}(C) \mid H^{0}(L \otimes M) \neq 0\right\} \\
& Y=\left\{M \in \operatorname{Pic}^{1}(C) \mid H^{0}\left(L^{\prime *} \otimes \omega_{C} \otimes M\right) \neq 0\right\} .
\end{aligned}
$$

Observe that $X$ is nonempty, since $\operatorname{deg}(L \otimes M)=\frac{d-s}{2}+1 \geq 0$, and that $Y$ is also nonempty because, by Serre duality, we can assume $d \leq 2 g-2$. Thus

$$
\begin{aligned}
\operatorname{deg}\left(L^{\prime *} \otimes \omega_{C} \otimes M\right) & =\frac{1}{2}(-d-s+4 g-4)+1 \\
& \geq \frac{1}{2}(-s+2 g-2)+1 \geq \frac{1}{2}(g-2)+1 \geq 0 .
\end{aligned}
$$

Observe also that

$$
\begin{aligned}
\operatorname{dim} X+\operatorname{dim} Y & =\operatorname{dim} W_{\frac{d-s}{2}+1}^{0}+\operatorname{dim} W_{-\frac{d}{2}-\frac{s}{2}+2 g-1}^{0} \\
& =\min \left\{\frac{d-s}{2}+1, g\right\}+\min \left\{-\frac{d}{2}-\frac{s}{2}+2 g-1, g\right\} \\
& \geq g=\operatorname{dim} \operatorname{Pic}^{1}(C) .
\end{aligned}
$$

Since, in the Chow ring of $\operatorname{Pic}^{1} C$, both $X$ and $Y$ are positive multiples of powers of the theta divisor, which is ample, this implies that $X$ and $Y$ have nonempty intersection. Let $M \in X \cap Y$ and let $A, B$ be effective divisors defined by nonzero sections of $L \otimes M, L^{\prime *} \otimes \omega_{C} \otimes M$. Let $\tilde{A}$ be the greatest common divisor of $A$ and $B$, and let $\tilde{B}=A+B-\tilde{A}$. We obtain, thus, the sequence

$$
0 \longrightarrow \mathcal{O}(\tilde{A}) \longrightarrow \mathcal{O}(A) \oplus \mathcal{O}(B) \longrightarrow \mathcal{O}(\tilde{B}) \longrightarrow 0 .
$$

Denoting $\tilde{L}=\mathcal{O}(\tilde{A}) \otimes M^{*}, \tilde{L}^{\prime}=L^{\prime} \otimes L^{*} \otimes \tilde{L}$, and $\tilde{E}=E \otimes L^{*} \otimes \tilde{L}$ we have sequences

$$
\begin{aligned}
& \left.0 \longrightarrow \tilde{L} \longrightarrow \tilde{E} \longrightarrow \tilde{L}^{\prime} \longrightarrow 0 \quad \text { (tensoring the above with } L^{*} \otimes \tilde{L}\right) \\
& 0 \longrightarrow \tilde{L} \longrightarrow L \oplus\left(L^{\prime *} \otimes \omega_{C}\right) \\
& \longrightarrow \tilde{L}^{\prime *} \otimes \omega_{C} \longrightarrow 0 \quad \text { (tensoring the above with } M^{*} \text { ) } \\
& 0 \longrightarrow \tilde{E} \longrightarrow E \oplus\left(E^{*} \otimes \omega_{C}\right) \\
& \longrightarrow \tilde{E}^{*} \otimes \omega_{C} \longrightarrow 0 \quad \text { (tensoring the former with } E \otimes L^{*} \text { ) }
\end{aligned}
$$

because $E^{*}=E \otimes L^{*} \otimes L^{\prime *}$ and $\tilde{E}^{*}=\tilde{E} \otimes \tilde{L}^{*} \otimes \tilde{L}^{\prime *}=E \otimes L^{\prime *} \otimes \tilde{L}$. 
Clearly $\tilde{E}$ has the same Segre invariant $s$ as $E$, and, since

$$
h^{0}(E)+h^{0}\left(E^{*} \otimes \omega_{C}\right) \leq h^{0}(\tilde{E})+h^{0}\left(\tilde{E}^{*} \otimes \omega_{C}\right),
$$

we will be done, by induction, if $s-2 \leq \operatorname{deg} \tilde{E}<\operatorname{deg} E$, because a twist $E_{s-2}$ of $E$ having degree $s-2$ has $h^{0}\left(E_{s-2}\right)+h^{0}\left(E_{s-2}^{*} \otimes \omega_{C}\right)=2 g-s$. This amounts to saying that $-1 \leq \operatorname{deg} \tilde{L}<\operatorname{deg} L$, which in turn is equivalent to $0 \leq \operatorname{deg} \tilde{A}<\operatorname{deg} A$. The first inequality is clear, but the second one amounts to saying that $A$ is not contained in $B$. Assume then, from now on, that $A$ is contained in $B$, so that $D=B-A \geq 0$ is the zero-divisor of a nonzero global section of $L^{*} \otimes L^{\prime *} \otimes \omega_{C}$. Since $\operatorname{deg} L \otimes M \geq n+1$, we can consider a line bundle $N$ of the same degree as $L \otimes M$ but having $h^{0}(N) \geq 2$.

Let $A_{2} \in|N|$ and write $B_{1}=D+A_{2}$. Now we can choose $A_{1} \in|N|$ not contained in $B_{1}$. As before, let $\tilde{A}_{1}$ be the greatest common divisor of $A_{1}$ and $B_{1}$, and $\tilde{B}_{1}=A_{1}+B_{1}-\tilde{A}_{1}$. We have then a sequence

$$
0 \longrightarrow \mathcal{O}\left(\tilde{A}_{1}\right) \longrightarrow \mathcal{O}\left(A_{1}\right) \oplus \mathcal{O}\left(B_{1}\right) \longrightarrow \mathcal{O}\left(\tilde{B}_{1}\right) \longrightarrow 0
$$

where $0 \leq \operatorname{deg} \tilde{A}_{1}<\operatorname{deg} A_{1}=\operatorname{deg} A$.

Denoting $\tilde{L_{1}}=\mathcal{O}\left(\tilde{A_{1}}\right) \otimes L \otimes N^{*}$, and $\tilde{L}_{1}^{\prime}=\tilde{L_{1}} \otimes L^{*} \otimes L^{\prime}$, and $\tilde{E}_{1}=$ $E \otimes L^{*} \otimes \tilde{L_{1}}$, we have sequences

$$
\begin{array}{ll}
0 \longrightarrow \tilde{L}_{1} \longrightarrow \tilde{E}_{1} \longrightarrow{\tilde{L_{1}}}^{\prime} \longrightarrow 0 & \text { (tensoring the above with } \left.L^{*} \otimes \tilde{L}_{1}\right) \\
0 \longrightarrow \tilde{L}_{1} \longrightarrow L \oplus\left(\tilde{L}^{\prime *} \otimes \omega_{C}\right) & \\
\longrightarrow \tilde{L}_{1}^{\prime *} \otimes \omega_{C} \longrightarrow 0 & \text { (tensoring the above with } \left.L \otimes N^{*}\right) \\
0 \longrightarrow \tilde{E}_{1} \longrightarrow E \oplus\left(E^{*} \otimes \omega_{C}\right) & \\
\longrightarrow \tilde{E}_{1}^{*} \otimes \omega_{C} \longrightarrow 0 & \text { (tensoring the former with } \left.E \otimes L^{*}\right)
\end{array}
$$

Here $-1 \leq \operatorname{deg} \tilde{L}_{1}<\operatorname{deg} L$, and thus the bundle $\tilde{E}_{1}$, of some invariant $s$ as $E$, has degree

$$
s-2 \leq \operatorname{deg} \tilde{E}_{1}=s+2 \operatorname{deg} \tilde{L}_{1}<s+2 \operatorname{deg} L=d .
$$

Therefore

$$
h^{0}(E)+h^{0}\left(E^{*} \otimes \omega_{C}\right) \leq h^{0}\left(\tilde{E}_{1}\right)+h^{0}\left(\tilde{E}_{1}^{*} \otimes \omega_{C}\right) \leq 2 g-s,
$$

which follows from the induction hypothesis, as above.

Finally, we prove the strict Severi bound for half of the cases when the bundle $E$ on the Riemann surface $C$ is general among those having the same invariant $s$.

Proposition 2.7. Let $E$ be a general rank two bundle of degree $d$ and Segre invariant $s \geq 0$ on a Riemann surface of genus $g \geq 1$. If $s \leq d \leq$ $4 g-4-s$, then

$$
h^{0}(E) \leq \frac{d-s}{2}+1
$$


Proof. It has been proved (Cor. 1) in case $s=g$. Let $s \leq g-1$. From the generality assumption, the bundle $E$ has an elementary transform $E^{\prime}$ at a general point $p \in C$,

$$
0 \longrightarrow E \longrightarrow E^{\prime} \longrightarrow \mathbb{C}(p) \longrightarrow 0
$$

with Segre invariant $s^{\prime}=s+1$. Since, by induction, we can assume that the bound holds for $E^{\prime}$, it also holds for $E$, as

$$
h^{0}(E) \leq h^{0}\left(E^{\prime}\right) \leq \frac{(d+1)-(s+1)}{2}+1 .
$$

\section{Severi bundles}

We give the name of Severi to the rank two bundles $E$ reaching the Severi bound with $\delta=2$, i.e. having

$$
h^{0}(E)=\frac{d-s}{2}+2 .
$$

By Serre's duality, we can assume $d \leq 2 g-2$. Then we only look for stable bundles since, because of Proposition 0.1, all the Severi bundles have $s \geq 0$, and the only Severi bundles with $s=0$ are $\mathcal{O}_{\mathcal{C}} \oplus \mathcal{O}_{C}\left(\right.$ or, dually, $\left.\omega_{C} \oplus \omega_{C}\right)$ on any curve $C$ and, if $d$ is a multiple of four, $E=L \oplus L$ for $L$ the hyperelliptic bundle of degree $d / 2$.

By Theorem 0.2, a necessary condition for a rank 2 bundle to be Severi is that the invariants $g, s, d$ satisfy $K_{\frac{d-s}{2}+1}(g, 2 g-s)=0$. Therefore, if there is a Severi bundle $E$ on a Riemann surface $C$ with invariants $g, s, d$, then it cannot be the case for the invariants $g, s, d-2$ or $d-4$. (Indeed, a corollary of the proof of Lemma 1.2 is that, for nonnegative integers $N \geq n, r$, we cannot have $K_{r}(n, N)=K_{r-1}(n, N)=0$. If furthermore $N \neq 2 n$, then it cannot be $K_{r}(n, N)=K_{r-2}(n, N)$, since this would imply $K_{r-1}(n, N)=0$.) Next we use the fact that $2 g-s \neq 2 g$ ), which implies that, for any $p, q \in C$, we have the identity $h^{0}(E(-p))=h^{0}(E)-2$ and $h^{0}(E(-p-q)) \leq h^{0}(E)-3$, because otherwise $E(-p)$ or $E(-p-q)$ would also be Severi bundles.

We conclude that the map $X \longrightarrow G r\left(h^{0}(E)-2, h^{0}(E)\right)$ (sending $x$ to the subspace of $H^{0}(E)$ consisting of sections vanishing at $x$ ) is in fact an immersion:

$A$ Severi bundle of invariants $g, s, d$ is always the restriction of the universal bundle of $\operatorname{Gr}\left(\frac{d-s}{2}, \frac{d-s}{2}+2\right)$ to a smooth curve of genus $g$ and degree $d$. It reduces the search for Severi bundles to a problem of curves in Grassmannians. 
By the Krawtchouk relation, a stable Severi bundle must have $d>s$. In Section 2 we provided families of invariants satisfying this relation, depending on the value of $d-s$. In this section we classify all the Severi bundles with $d-s=2,4$. Recall that, for each $2 k=d-s \geq 6$, there are only finitely many triples $(g, s, d)$ for which Severi bundles are expectable. The identification of these "sporadic bundles" is left as an open problem.

Case $d-s=2$. Since the family $F_{1}$ is complete (Lemma 2.2), the "Krawtchouk relation" for triples outside the boundary holds if and only if $g=\frac{(d-1)(d-2)}{2}$. The only Krawtchouk triple of the boundary with $d-s=2$ is the Serre-self-dual triple $(3,2,4)$, which also has $g=\frac{(d-1)(d-2)}{2}$. Therefore, all Severi bundles with $d-s=2$ fall among those described in the following

Proposition 3.1. A rank two stable bundle $E$ of degree $d \leq 2 g-2$ and Segre invariant $s=d-2$ on a curve $C$ of genus $g=\frac{(d-1)(d-2)}{2}$ is a Severi bundle if and only if $C$ is a plane curve and $E$ is the restriction of $T_{\mathbb{P}^{2}}(-1)$.

Proof. From what we have noted, such a Severi bundle must be the restriction of the universal bundle $T_{\mathbb{P}^{2}}(-1)$ of $\operatorname{Gr}(1,3)=\mathbb{P}^{2}$ to a smooth curve of degree $d$. Let us see that, conversely, the restriction $E=\left.T_{\mathbb{P}^{2}}(-1)\right|_{C}$ for a smooth plane curve of degree $d$, thus with $\operatorname{deg} E=d$ and $g=\frac{(d-1)(d-2)}{2}$, has Segre invariant $s=d-2$ and $h^{0}(E)=3$, i.e. it is a Severi bundle. Restricting to $C$ the universal sequence of $\mathbb{P}^{2}$, we get $0 \longrightarrow \mathcal{O}_{C}(-1) \longrightarrow V \otimes \mathcal{O}_{C} \longrightarrow T_{C}(-1) \longrightarrow 0$ (where $V=H^{0}\left(\mathcal{O}_{\mathbb{P}^{2}}(1)\right)^{*}$ ); thus $h^{0}(E) \geq 3$, and in fact, by our Severi bound, $h^{0}(E)=3$.

The assertion $s \leq d-2$ is trivial since, for any $x \in C$, the bundle $E(-x)$ has degree $d-2$ and $h^{0}(E(-x)) \geq h^{0}(E)-2>0$. David Butler asserts in [3, Prop. 2.1], that in fact $s=d-2$ because $E(-x)$ has infinitely many line subbundles of maximal degree. This is certainly true, although we do not see why it is, in itself, a reason to conclude that $s \geq d-2$. David Butler has been more explicit with the following argument, which he has gently communicated to us, showing that all line subbundles

$$
0 \longrightarrow L \longrightarrow E \longrightarrow L^{\prime} \longrightarrow 0
$$

of $E$ have $\operatorname{deg} L \leq 1$ (i.e. that $s \geq d-2$, as wanted): Let $W$ be the image of $V=H^{0}(E) \longrightarrow H^{0}\left(L^{\prime}\right)$, and denote by $F$ the bundle in

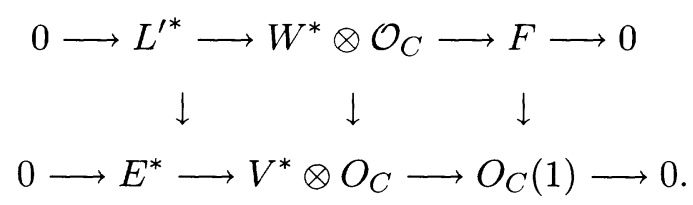

Observe that $F$ cannot have torsion since the snake morphism would send it into the torsion free sheaf $E^{*} / L^{\prime *}$. We distinguish two cases: 
If $\operatorname{dim} W \leq 2$, then in fact $\operatorname{dim} W=2$, because $\operatorname{dim} W=1$, i.e. $F=0$, would imply $L^{\prime *}=\mathcal{O}_{C}$, contradicting $h^{0}\left(L^{\prime *}\right) \leq h^{0}\left(E^{*}\right)=0$, since $V^{*}=$ $H^{0}\left(\mathcal{O}_{\mathbb{F}^{2}}(1)\right) \hookrightarrow H^{0}\left(\mathcal{O}_{C}(1)\right)$. In other words, $F$ is a line bundle on a smooth plane curve $C$ of degree $d$, having $h^{0}(F) \geq 2$. In [1, p. 56], this implies $\operatorname{deg} F \geq d-1$ which means $\operatorname{deg} L^{\prime *} \leq 1-d$, and thus $\operatorname{deg} L \leq 1$, as wanted.

If $\operatorname{dim} W=3$, then $F$ is a rank two bundle, with $h^{0}(E) \geq 3$ generated by global sections; thus a general section

$$
0 \longrightarrow \mathcal{O}_{C} \longrightarrow F \longrightarrow \operatorname{det} F \longrightarrow 0
$$

is nowhere vanishing, providing a line bundle $\operatorname{det} F$ with $h^{0}(F) \geq 2$, which again implies $\operatorname{deg} F=\operatorname{deg}(\operatorname{det} F) \geq d-1$, thus concluding $\operatorname{deg} L \leq 1$.

Case $d-s=4$. Besides Krawtchouk triples in the boundary, those with $d-s=4$ are described as family $F_{2}$ in Lemma 2.2 , i.e. as those having $d \not \equiv 1$ $(\bmod 3), 9 \leq d<2 g-2$, and $g=\frac{(d-3)(d-2)}{6}$. We will show these do not actually correspond to Severi bundles. On the other hand, in case $d-s=4$, only the triples $(g, 0,4)$ and the dual ones $(g, 2 g-6,2 g-2)$ are in the boundaries. Since we only have to consider $0<s<g$ (because of the introduction of this section and of Corollary 1$)$, these reduce to the one case $(5,4,8)$ which has $d \not \equiv 1$ $(\bmod 3)$ and $g=\frac{(d-2)(d-3)}{6}$. In [9] these cases were shown to exist. Now we are in position to find all such bundles.

Proposition 3.2. The only Severi bundles with degree $d \leq 2 g-2$, and Segre invariant $s=d-4$ are the stable restrictions of the universal quotient bundle of $G=\operatorname{Gr}(2,4)$ to smooth irreducible curves $C$ of bidegree $(6,2)$ in special linear complexes.

We recall that a special linear complex is a three-dimensional cone $C_{3}$ in $G$, i.e. the intersection of $G \subseteq \mathbb{P}^{5}$ with a tangent $\mathbb{P}^{4}$, which corresponds to all lines of $\mathbb{P}^{3}$ meeting a given line. It contains a pencil of $\alpha$-planes and another pencil of $\beta$-planes: an $\alpha$-plane of $G$ consists of all lines of $\mathbb{P}^{3}$ passing by a given point, and a $\beta$-plane consists of all lines of $\mathbb{P}^{3}$ contained in a given plane. A line of $G$ consists of all lines of $\mathbb{P}^{3}$ contained in a plane and passing by a point of it, so that it is contained in exactly one $\alpha$-plane and one $\beta$-plane. A curve $C$ of a special linear complex $C_{3}$ has bidegree $\left(\delta, \delta^{\prime}\right)$ if it meets the $\alpha$-planes of $C_{3}$ in $\delta$ points, counted with multiplicity, and the $\beta$-planes in $\delta^{\prime}$ points. Since the union of an $\alpha$-plane and a $\beta$-plane of $C_{3}$ is a hyperplane section, the degree of $C$ is $d=\delta+\delta^{\prime}$.

Proof of Proposition 3.2. We know $g=\frac{(d-2)(d-3)}{6}$ and $d \not \equiv 1 \quad(\bmod 3)$. We can assume $g \geq 4$ (because $0<s=d-4 \leq 2 g-6$ ), and thus $d \geq 8$. Because of an observation made at the beginning of this section, such a Severi bundle $E$ is the restriction of the universal bundle $\mathcal{U}$ of $G=\operatorname{Gr}(2,4) \subseteq \mathbb{P}^{5}$ to a curve $C$ 
of degree $d$ and genus $g=\frac{(d-2)(d-3)}{6}$. The fact that $E$ has no nonzero sections vanishing at three points can be interpreted as the nonexistence of $\beta$-planes trisecant to $C$. This implies that $C$ is degenerate, i.e. contained in $\mathbb{P}^{4}$. On the other hand $C$ is not contained in $\mathbb{P}^{3}$ because it is not contained in a quadric surface $Q_{2}$, since $\left.\mathcal{U}\right|_{Q_{2}}$ splits as $\mathcal{O}_{Q_{2}}(1,0) \oplus \mathcal{O}_{Q_{2}}(0,1)$, and thus $E$ would be otherwise decomposable. Nor could it be contained in a quadric cone, because then $g$ would be $\frac{(d-2)^{2}}{4}$ or $\frac{(d-1)(d-3)}{4}$ according to its parity; nor could it be contained in a plane. Therefore $C$ is a Castelnuovo curve of $\mathbb{P}^{4}$, thus contained in the complete intersection of a cubic scroll with a hypersurface of degree $\frac{d+1}{3}$ if $d \equiv 2 \quad(\bmod 3)\left(\frac{d}{3}\right.$ if $\left.d \equiv 0\right)$, the "rest" consisting of just generators of the scroll or generators plus one unisecant. Therefore the generators of the scroll meet $C$ in $\frac{d+1}{3}$ or $\frac{d-2}{3}$ points $\left(\frac{d}{3}\right.$ or $\frac{d}{3}-1$ points, if $d \equiv 0$ ) (cf. for instance, [7, pp. 527-533]). This scroll must be contained in the three-dimensional quadric $\mathbb{P}^{4} \cap G \subseteq \mathbb{P}^{5}$; otherwise $C$ would be of degree at most 6 . Each generator of the scroll, being a line of $G$, is contained in a $\beta$-plane, so we get $\beta$-planes meeting $C$ at $\frac{d+1}{3}$ or $\frac{d-2}{3}$ points, i.e. sections of $E$ vanishing at so many points; thus this number is at most 2 , and $d=8$. If this quadric were smooth, all its surfaces would be complete intersections, thus of even degree; so it is a special linear complex $C_{3}$.

Let $\left(\delta, \delta^{\prime}\right)$ be the bidegree of $C$ in $C_{3}$, thus with $\delta+\delta^{\prime}=8$ and $\delta^{\prime} \leq 2$. If $\delta^{\prime}=1$, then $C$ cannot intersect a generator of $C_{3}$ in more than one point, since it is contained in a $\beta$-plane, so that $C$ projects isomorphically, from the vertex of $C_{3}$, to a $(7,1)$ curve of the smooth quadric surface $Q_{2}$, contradicting the fact that $C$ is not rational. Therefore $C$ has bidegree $(6,2)$ as stated.

Conversely, let $C$ be a curve a genus 5 and bidegree $(6,2)$ in a special linear complex, having stable restriction $E$ of the universal bundle. Such curves exist because we know, after [9], that Severi bundles with $(g, s, d)=(5,4,8)$ must exist. Let $S$ be the ruled surface of $\mathbb{P}^{3}$ corresponding to $C$, so that its desingularized ruled surface $\tilde{S}$ is $\mathbb{P}(E)$ for the restriction $E$ of the universal bundle to $C$. Then $4=h^{0}\left(\mathcal{O}_{\mathbb{P}^{3}}(1)\right) \leq h^{0}\left(\mathcal{O}_{\tilde{S}}(1)\right)=h^{0}(E)$ and, as shown in [9], this implies $s=4$.

According to [9] the general curve of genus 5 has a Severi bundle with $s=4, d=8$; thus it occurs among those described in Proposition 3.2.

Universidad Autónoma de MAdRid, 28049 Madrid, Spain

E-mail address: franciscojavier.cilleruelo@uam.es

Universidad Complutense de MAdRID, 28040 Madrid, SPAin

E-mail address: sols@mat.ucm.es 


\section{REFERENCES}

[1] E. Arbarello, M. Cornalba, P. A. Griffiths, and J. Harris, Geometry of Algebraic Curves, Grundlehren Math. Wiss. 267, Springer-Verlag, New York (1985).

[2] E. Arrondo AND I. Sols, Bounding sections of bundles on curves, London Math. Soc. Lecture Note Series 179 (Trieste, 1989/Bergen, 1989), 24-31, Cambridge Univ. Press, Cambridge, 1992.

[3] D. Butler, Families of maximal subbundles for rank two bundles on a curve, Math. Ann. 307 (1997), 29-39.

[4] D. Eisenbud AND J. Harris, Divisors on general curves and cuspidal rational curves, Invent. Math. 74 (1983), 371-418.

[5] - A simpler proof of the Gieseker-Petri theorem on special divisors, Invent. Math. 74 (1983), 269-280.

[6] W. Fulton and R. Lazarsfeld, On the connectedness of degeneracy loci and special divisors, Acta Math. 146 (1981), 271-283.

[7] P. Griffiths and J. Harris, Principles of Algebraic Geometry, John Wiley \& Sons, Inc., New York (1978).

[8] - On the variety of special linear systems on a general algebraic curve, Duke Math. J. 47 (1980), 233-272.

[9] I. GrzegorczyK, All bundles in $W_{2,8}^{3}$ have infinitely many maximal subbundles, Ulam Quart. 3 (1996), 3pp.

[10] G. HANRot, Résolution effective d'équations diophantiennes: algorithmes et applications, Thèse, Université Bordeaux 1 (1997).

[11] R. Hartshorne, Algebraic Geometry, Grad. Texts in Math. 52, Springer-Verlag, New York (1977).

[12] T. JoHnsEn, Rank two bundles on algebraic curves and decoding of Goppa codes, preprint.

[13] I. KRASIKov and S. Litsyn, On integral zeros of Krawtchouk polynomials, J. Combin. Theory 74 (1996), 71-99.

[14] H. Lange and M. S. Narasimhan, Maximal subbundles of rank two vector bundles on curves, Math. Ann. 266 (1983), 55-72.

[15] M. Mignotte and A. Pethő, On the system of diophantine equations $x^{2}-6 y^{2}=-5$ and $x^{2}=2 z^{2}-1$, Math. Scand. 76 (1995), 50-60.

[16] M. Nagata, On self-intersection number of a section on a ruled surface, Nagoya Math. J. 37 (1970), 191-196.

[17] F. Severi, Sulla clasificazione delle rigate algebrice, Rend. Mat. 2 (1941), 1-32.

[18] R. J. Stroeker and B. M. M. De Weger, On a quartic Diophantine equation, Proc. Edinburgh Math. Soc. 39 (1996), 97-114.

[19] - On integral zeroes of binary Krawtchouk polynomials, Nieuw Arch. Wisk. 17 (1999), 175-186.

[20] Solving elliptic diophantine equations: the general cubic case, Acta Arith. 87 (1999), 339-365.

[21] X. J. TAN, Extensions of Clifford's theorem (Chinese), Acta Math. Sinica 31 (1988), $710-720$.

[22] A counterexample of Arrondo-Sols' conjecture, Chinese Sci. Bull. 37 (1992), 1145-1149.

[23] M. Teixidor i Bigas, Brill-Noether theory for stable vector bundles, Duke Math. J. 62 (1991), 385-400.

(Received August 28, 2000) 